THAT WILL ENHANCE APPLICATIONS TO ENGINEERING DESIGN

\author{
John J. Allan III \\ Associate Professor of Mechanical Engineering \\ and of Computer Sciences \\ Director, Computer Applications Laboratory \\ The University of Texas at Austin \\ Austin, Texas 78712
}

SUMMARY

This paper describes some research in man/machine interaction, and graphics hardware/software that will enhance applications to engineering design. Research aspects of CAD executive systems, command languages, and networking are mentioned. Finally, a few areas where little or no research is being done are identified, and consideration is called for.

INTRODUCTION

This paper talks about research advances in computer graphics. This is not a paper on computer-aided design. The word research here means technical achievements that are not yet completed or commercially available. This paper is not intended to be exhaustive. In fact, it is really meant to stimulate thinking about things that have been and should be done. It is the author's feeling that more research needs to be done in computer graphics so that it can better and more economically enhance engineering design.

Further, these "advances" are discipline independent. Some of them relate to $\mathrm{man} / \mathrm{machine}$ interaction, some relate to new hardware/software combinations, while still others refer to data structuring for graphic presentation, command languages for graphic input and output, and the design of executive systems and networks, especially as related to computer graphics.

\title{
MAN/MACHINE INTERACTION
}

Many of the things that we do when we design and build interactive graphic displays for use in engineering design are done by what we call "gut feel." This is fine, and in many cases it may even be accurate. However, the increasing sophistication of both the CAD system behind the graphic display, and indeed, the graphic display itself, demands that we have solid data on which to base the decisions about the design of the software and hardware for our displays. 
Consequently, work has been done to substantiate, or disprove, certain ideas or concepts that many engineering design practitioners have had about interactive graphics. In fact, this paper is, in part, a call for a much greater effort in this area. The reason for that is that in the not-too-distant future, as our colleagues in artificial intelligence and pattern recognition make greater and greater advances, and as computing equipment becomes even less expensive, it will be possible to have CAD systems which adapt to the individual design engineer. When this comes about, it is going to be extremely important for us to be able to design those systems so that when they adapt to a new designer, they adapt in a most effective way. That will give the designer the greatest freedom for making decisions in the design process.

One study completed in 1971 and reported in 1973 (1)* by Corley, reported for the first time a statistically significant experiment in which a large group of design engineers were asked to route some piping through a factory such that the thermal-stresses would be minimized. These engineers were asked to work on problems of four levels of complexity. Furthermore, each one was asked to route the pipe through the plant topologically in a keyboard mode, in a drawing mode through a tablet in which the drawing was drawn to scale, and then in a third mode, which was a combination of these in which the pipe-routing topology was entered by drawing but the dimensions were entered by keyboard. The point was to verify the fact that topologically entering the pipe-routing by drawing is not only a significantly quicker way to enter the data than by keyboard, but is less error prone. Also, it was desired to investigate the learning rates of these design engineers as they went to consecutively tougher problems using this new equipment. Their learning rates did show up in the data.

In subsequent and more substantial pieces of work, Corley reports using pragmatic information content as an aid in the design of interactive graphic displays.

In one report Corley (2) reports on the specification of the pragmatic information content of graphical displays as approached by defining three display attributes: quantity, format, and time. He proposes a mathematical measure of the information quantity in the display, and discusses qualitatively the effects of format and time on pragmatic information processing. He presents data from an experimental evaluation of the effects of quantity, format, and time on pragmatic information processing in a specially constructed graphically-oriented engineering task.

In another publication, Corley (3) develops the pragmatic information content of a "frame" in an interactive graphic display. He also presents in that publication an example of cam design, and goes into detail as to the predicted quantity of information in the display.

${ }^{\star}$ Parentheses refer to numbered references at the end of the paper. 
In another study, Beazley (4) discusses the evaluation of interactive graphics used in the engineering design environment. He presents a general theory of design behavior, and the essential elements of design communication are then reduced to the study of digraph messages. The study relates to the construction and editing of models of engineering systems done by selecting nodes and specifying their relationship to each other with arcs. He presents the experimental results of a study of digraphs, their formats, and the associated learning tasks. This is all presented as a study of computer graphics acceptability for engineering design use.

In the Beazley study the formats of the screen were chosen to evaluate the special features of interactive graphics which distinguish it from highly non-spatial alpha/numeric terminals. The results indicated that digraphs could be learned with significantly less learning errors when the subjects were permitted to use the spatial cues available in interactive graphics. Other results are included and discussed.

A current study is underway which will use a group of design engineers to evaluate the effect of audio feedback on problems in which one of the cues to the designer in the real world would be audio feedback. This is applicable to many problems such as tolerances in mechanical linkages, the mounting of loud speakers in audio equipment, and for the particular problem chosen in this study, the design of an acoustically treated computer-aided design laboratory. Subjects will be asked to design an acoustically treated CAD laboratory to optimize the noise levels for the design engineers that will be working there. As the experimental subjects sit at the CAD terminal designing the various configurations of the room, the D-A equipment will be driving noise generators, which will feed back to them audio signals simulating the noise level of the room that they are designing. Control groups are being held that are not being given the audio feedback so that the effect of the audio feedback in achieving the design criteria can be measured.

It is in this author's future plans, when the equipment becomes available, to run controlled studies of audio input coupled with interactive graphics. A first paper in this area was presented by Griffith and Riganati (5) in which they discuss an interactive graphics system with bi-directional audio capability. It has been used for classifying and describing speech and image data. While not slanted specifically to engineering design, it has been used extensively by signal processing and pattern recognition groups to perform research and advance development studies in speech, image, specialized low-cost optical character recognition, and fingerprint processing.

\section{NEW HARDWARE/SOFTWARE DEVELOPMENTS}

The video frame buffer and some of its associated software as described by Kajiya, and Catmull $(6,7)$ is a new computer graphics facility for displaying three-dimensional objects. Probably as important, or more important than the hardware frame buffer itself and its 
associated technological accomplishments, is the thinking that has been going on about the way to store data which can subsequently be transformed to represent three-dimensional objects. The papers referenced here describe a random-access semi-conductor memory and discuss the central design considerations necessary to drive a shaded picture television display. Also presented in these references is a method for producing computer-shaded pictures of curved surfaces, including the notion of mapping onto patches of those surfaces, texture for computer-generated pictures.

In other publications Laws, and Sproull and Newman $(8,9)$ describe gray-scale graphic processing from both the hardware and software points of view on raster-scan graphic displays. These papers explain the main reasons for choosing to build a run-code gray-scale graphic processor, and indicate some of the problems in the design. Also included is the description of a graphics package for use with raster-scan displays. This latter involves an extension to conventional techniques for constructing general-purpose line-drawing graphics software for the definition and display of solid opaque objects.

In a paper by Denes (10), he describes a computer-controlled scan display system specially designed for interactive applications. A particular feature of the system is that the color of each display element is under program control.

Because high-speed interaction with a conventional refresh graphic system is most desirable, this author and one of his colleagues have just installed 16-bit parallel 50-kilobaud interfaces from a PDP-11/40 to their four Imlacs. This hardware is in part described by Stowell (11) in an article in which he describes the architecture of an interactive three-dimensional random vector display processor. Some of the realtime capabilities provided by the hardware described in the paper are picture rotation, translation, scaling, perspective projection, manual picture manipulation, programmable intensities and line-types, selective editing, mirror imaging, and windowing.

Other graphic devices such as the laser-scan display developed at Cambridge are all contributing to the further enhancement of the picture that can be presented to the design engineer.

The point of including the above references to new hardware and software is that while they may be argued out of the picture for economical engineering design in 1975, they are not as drastic a departure from what we are doing today, as Sketch-Pad was, from what we were doing in 1963. The point here is that it is incumbent upon engineering designers and CAD professionals to learn how to best take advantage of the computer graphics capabilities that can act as a most natural front-end to a computer-based engineering design system. It appears that the real hang-up is how to structure the data. Regardless of whether the data are going to be handled by hardware or software, how to structure them is not adequately known. 
To that end, Allan (12) calls for further work on formal data-base descriptions so that transformers that yield graphic post conditions can be applied. It is well-known that engineering designers would like to be able to: (1) free-hand sketch for "feel," (2) prescribe the topology of a reticulated physical system, (3) enter complex surfaces that describe physical property data, (4) handle imprecise or qualitative data, (5) connote "solidness," (6) plot 3-D physical objects with grayscales, (7) provide output animation, (8) "recognize" digitized pictures of prior designs, (9) etc. It is trying to get all of these representations from the same data that is forcing the need for new and better graphics hardware, software, and data structuring techniques.

\section{OTHER RELEVANT CONCERNS}

Three other major relevant concerns have a bearing on computer graphics as related to engineering design. Developments in CAD executive systems, in computer graphic command languages, and in computer graphic networking are all relevant areas.

The importance of CAD executive systems, as they relate to research advances in computer graphics that will enhance applications to engineering design, is that in the overall design of a CAD system the type of graphics and the use that it will be put to has to be integrated with the design and capability of the entire CAD system. Further, this must be considered in terms of the environment in which it is going to be used. So to this end, a great deal of research needs to be done on functioning CAD systems to find out the relative effectiveness with which types of graphics devices and the methods in which their messages are formatted can increase the effectiveness of the design engineer.

The area of command languages is of vital importance because being able to couple the type of computer graphic facilities with the appropriate ability to communicate concepts and ideas will give rise to the more cost-effective utilization of computer graphics in engineering design. This will continue to be a problem as the latest graphics equipment available is much more expensive than what is called conventional engineering design graphics as generally reported in this conference.

It is apparent to most practitioners in CAD that it is going to soon become economically unviable for everyone to generate his own software. Therefore, parts and pieces of software are going to have to be shared from distant locations. Because the type of graphic device dictates the protocol for bringing in messages, the relationship to networking is going to be important. That fact is prompting the IIASA/IFIP W.G. 5.2 task group on International CAD Networking to look into standards, 
protocols, etc. A paper in this area by Cohen (13) discusses the network issues of computer graphics over a general purpose digital computer communication network. It is pointed out in the article that the hugh variety of graphics equipment and general purpose computers is the real problem to solve. The network transmission issues are well-understood and generally solved. It goes on to point out that non-trivial effects of a network on graphics are band width, error-control, and delay issues. This will be a particularly greater problem as corporations and government institutions with diverse design groups want to begin to communicate on an integrated CAD system coupled by networking. How we design graphics and how we use graphics for engineering design in that environment will take a great deal more research.

\section{NOW AND IN THE FUTURE}

When Hatvany, Newman, and Sabin (14) were commissioned by IIAS to do a world survey of computer-aided design, one of the areas to which they paid special attention was computer graphics. The reason that this paper argues that a great deal of research needs to be done in the area of computer graphics is that we need to turn computer power on itself in the area of applications much as the manufacturers have done in the area of manufacturing. We need to be able to use higher-level recognition of symbols, concepts, and ideas in order to make the conceptual input of computer graphics as applied to engineering design realizable. The argument that is always used against us is that computer graphics is too expensive. To quote from the world survey of computer-aided design: "Overall, our survey of the use of computer graphics in CAD leaves us to make the following observations: (1) we have found a great interest in 'turn-key' graphics systems, particularly among the electronics firms. These systems enable the user to ignore the programmer's problems and also permit the design of graphical 'front-ends' to existing batch operated programs. (2) Computer graphic equipment is expensive to buy and expensive to use. These are less serious problems now than they once were, but we will see great hesitation to use graphics in CAD because of the cost. (3) Designers of graphic software are reluctant to try new techniques partly because of ignorance and partly because of programming difficulty. This prevents economic use of computer graphics. Techniques we would like to see more widely used are procedure-driven rather than data-structure-driven graphic output, display files used for the update of storage tube displays, and on-line character recognition as an input technique. (4) Graphic hardware is rarely designed with the programmer in mind and graphic system hardware shows singular lack of understanding of the application programmers needs. There is a need for simpler hardware and more flexible software. We have seen signs of progress in these directions. (5) It is appropriate to consider drawing up standards for the functions to be provided by graphics packages. IIASA should maintain contact with the SIGGRAPH graphic standards planning committee with a view to benefitting for many standards or definitions that this committee produces." 
In light of the last section of their observations, IFIP W.G. 5.2 has also developed a committee which is in liaison with the SIGGRAPH graphics standards planning committee to develop a set of graphics standards that will probably reflect to a great extent those things found in GINO-F and other similar graphics packages.

Another area of graphics research has to do with the social ramifications of using computer graphics in the area of engineering design. Some of the many questions that we should address ourselves to relate to what happens when we use computer graphics to increase the effectiveness of engineering designing systems. If we displace people in their communities, if we give increased leverage to large industries that can afford sophisticated CAD systems, if we spawn a group of small specialist industries, or if we subsequently lose certain mid-size concerns, what advance planning should we do?

Another aspect that we should consider in the area of engineering design is: what should be changed in education as computer graphics is proliferated and becomes evermore economically viable? On the other hand, there are questions which are even further out in the future. For instance, computer graphics gives us a new way to communicate to analysis programs like we have never had before. And as computer graphics becomes more viable, and is coupled to even larger-scale computation, it may be that we will be able to design products that the average person cannot comprehend. What is the social consequence of that action?

\section{CLOSING REMARKS}

The whole idea of research into computer graphics as it is related to engineering design comes back to the fact that we are in a technologicallybased society. For that reason, to keep improving our lot, we design what we collectively feel are new and better things. Because of the competition of free enterprise and other pressures, we would like to design ever more comprehensively and ever-faster. This has driven us to find a syntax with which we can convey ideas, concepts, and trends without resorting to some complicated syntax to communicate with large data bases and large computational schema. The drive for this is economics. The resistance in the engineering community to computer graphics is purely economic, which includes the educational aspect. So, from that point of view, we need to plan wisely and do research so that we are not always flying just on the "gut feel" that we have about the goodness or the badness of graphics. To that extent this paper then is a call for more studies on man/machine interaction and a call for cooperation in the integration of the latest in graphics hardware and software into a pragmatic CAD environment so that the effects of the new hardware can be measured. All the time, this should be done by people who realize that they are responsible professionals in a total environment and therefore will also incorporate research into the social implications of our ability to communicate ever-faster and ever more comprehensively with engineering design systems. 


\section{REFERENCES}

1. Corley, Melvin R., and John J. Allan III; "The Effectiveness of Direct Graphical Entry of Topological and Geometric Data," Journal of Behavior Research Methods and Instrumentation, Vol. 5, No. 2, 1973, pp. 197-199.

2. Corley, M. R., and John J. Allan III; "Pragmatic Information Processing Aspects of Graphically-Accessed Computer-Aided Design," Proceedings of IEEE 1975 International Conference on Cybernetics and Society, WeAM4, September 23-25, 1975, San Francisco.

3. Corley, M. R.; "Use of a Pragmatic Information Measure as an Aid in the Design of Interactive Graphic Displays," ASME Publication 75-DET-88, 1975.

4. Beazley, William G.; "Man-Machine Communication of the Structure of Engineering Design Problem Information," MS Thesis, Department of Mechanical Engineering, The University of Texas at Austin, 1974.

References $\underline{5}$ through 13 are a 11 to be found in Proceedings of the Conference on Computer Graphics, Pattern Recognition, and Data Structure, IEEE \#75CH0981-1C, May 14-16, 1975.

5. Griffith, M. L. and J. P. Riganati; "Interactive Audio-Graphics for Speech and Image Characterization," pp. 163-169.

6. Kajiya, James T., Ivan E. Sutherland, and Edward C. Cheadle; "A Random-Access Video Frame Buffer," pp. 1-6.

7. Catmu11, Edwin; "Computer Display of Curved Surfaces," pp. 11-17.

8. Laws, B. A.; "A Gray-Scale Graphic Processor Using Run-Length Encoding," pp. 7-10.

9. Sproull, Robert F., and William M. Newman; "The Design of Gray-Scale Graphics Software," pp. 18-20.

10. Denes, Peter B.; "A Scan-Type Graphics System for Interactive Computing," pp. 21-24.

11. Stowe11, Garrett W., and Richard E. Garrett; "A Three-Dimensional Display Processor Design," pp. 157-162. 
12. Allan, John J.; "It's Time for a Different View of Data Structures for Computer Graphics," pp. 309-311.

13. Cohen, Dan; "Computer Graphics Over Computer Networks," pp. 294-296.

14. Hatvany, Joseph, William M. Newman, and Malcolm Sabin;

Report to IIASA on a World Survey of Computer-Aided Design, International Institute for Applied Systems Analysis, Laxenburg, Austria, July 1974, 52 pages. 